\title{
Load Balanced Routing Mechanisms for Mobile Ad Hoc Networks
}

\author{
Amita RANI ${ }^{1}$, Mayank DAVE ${ }^{2}$ \\ ${ }^{1}$ Department of Computer Science \& Engineering, University Institute of Engineering \& Technology, Kurukshetra, India \\ ${ }^{2}$ Department of Computer Engineering, National Institute of Technology, Kurukshetra, India \\ Email:amita26@rediffmail.com \\ Received May 11, 2009; revised July 11, 2009; accepted August 24, 2009
}

\begin{abstract}
Properties of mobile ad hoc networks (MANET) like dynamic topology and decentralized connectivity make routing a challenging task. Moreover, overloaded nodes may deplete their energy in forwarding others packets resulting in unstable network and performance degradation. In this paper we propose load-balancing schemes that distribute the traffic on the basis of three important metrics - residual battery capacity, average interface queue length and hop count along with the associated weight values. It helps to achieve load balancing and to extend the entire network lifetime. Simulation results show that the proposed load-balancing schemes significantly enhance the network performance and outperform one of the most prominent ad hoc routing protocols AODV and previously proposed load balanced ad hoc routing protocols including DLAR and LARA in terms of average delay, packet delivery fraction and jitter.
\end{abstract}

Keywords: Load Balanced Routing, Residual Battery Capacity, Hop Count, DLAR, LARA

\section{Introduction}

The proliferation of devices that do not depend upon centralized or organized connectivity has led to the development of mobile ad hoc networks (MANETs). These are the infrastructure-less networks where each node is mobile and independent of each other. Due to unorganized connectivity and dynamic topology, routing in MANET becomes a challenging task. Moreover, constraints like lower capacity of wireless links, error-prone wireless channels, limited battery capacity of each mobile node etc., degrade the performance of MANET routing protocols. Heavily-loaded nodes may cause congestion and large delays or even deplete their energy quickly. Therefore, routing protocols that can evenly distribute the traffic among mobile nodes and hence can improve the performance of MANETs are needed.

Routing protocols in MANETs are classified into three categories: proactive, reactive and hybrid routing protocols. Most of the prominent routing protocols like AODV [1], DSR [2] use hop count as the route selection metric. But it may not be the most efficient route when there is congestion or bottleneck in the network. It may lead to undesirable effects such as longer delays, lower packet delivery fraction and high routing overhead. Also some nodes that may lie on multiple routes spend most of their energy in forwarding of packets and deplete their energy quickly. Consequently they leave the network early. In this paper we present novel load-balancing mechanisms/schemes for MANETs that focus on distributing the traffic on the basis of combination of following three metrics:

- hop count

- residual battery capacity and

- average number of packets queued up in the interface queue of a node lying on the path from source to destination/traffic queue.

These three metrics along with associated weight values decide the path to be selected for data transmission. The results of simulations indicate that the proposed schemes outperform a prominent ad hoc routing protocol AODV and previously proposed load balanced ad hoc routing protocols including DLAR [3] and LARA [4] in terms of average delay, packet delivery fraction and jitter.

The rest of this paper is organized as follows. Section 2 discusses the work related to currently proposed load balanced ad hoc routing protocols. Section 3 details the proposed schemes in order to balance the load on various routes. Section 4 describes the methodology, performance metrics used and simulation results. Finally Section 5 concludes the paper. 


\section{Related Work}

Load balanced routing aims to move traffic from the areas that are above the optimal load to less loaded areas, so that the entire network achieves better performance. If the traffic is not distributed evenly, then some areas in a network are under heavy load while some are lightly loaded or idle. There are various proposed algorithms for load balanced routing. In Dynamic Load Aware Routing (DLAR) protocol [3] routing load of a route has been considered as the primary route selection metric. The load of a route is defined as the summation of the load of nodes on the route, and the load of a node is defined as the number of packets buffered in the queue of the node. To utilize the most up-to-date load information when selecting routes and to minimize the overlapped routes, which cause congested bottlenecks, DLAR prohibits intermediate nodes from replying to route request messages.

Another network protocol for efficient data transmission in mobile ad hoc networks is Load Aware Routing in Ad hoc (LARA) [4] networks protocol. In LARA, during the route discovery procedure, the destination node selects the route taking into account both the number of hops and traffic cost of the route. The traffic cost of a route is defined as the sum of the traffic queues of each of the nodes and its neighbors and the hop costs on that particular route. Thus, the delay suffered by a packet at a node is dependent not only on its own interface queue but also on the density of nodes. In routing with load balancing scheme (LBAR) [5], the destination collects as much information as possible to choose the optimal route in terms of minimum nodal activity (i.e the number of active routes passing by the node). By gathering the nodes activity degrees for a given route the total route activity degree is found. Load Sensitive Routing (LSR) protocol [6] is based on DSR. In LSR the load information depends on two parameters: total path load and the standard deviation of the total path load. Since destination node doe not wait for all possible routes, the source node can quickly obtain the route information and it quickly responds to calls for connections. Correlated Load-Aware Routing (CLAR) [7] protocol is an on-demand routing protocol. In CLAR, traffic load at a node is considered as the primary route selection metric and depends on the traffic passing through this node as well as the number of sharing nodes. Alternate Path Routing (APR) protocol [8] provides load balancing by distributing traffic among a set of diverse paths. By using the set of diverse paths, it also provides route failure protection. Reference [9] gives a comparative study of some of the load balanced ad hoc routing protocol.

All The protocols discussed above concentrate on traffic balancing and do not emphasize on energy issues. A number of routing protocols that consider energy issues in MANETs have been proposed. On the basis of route selection criterion, there are mainly two categories of the energy efficient routing protocols. The first class [10-12] selects the path that consumes the least energy to transmit a single packet from source to destination, aiming at minimizing the total energy consumption along the path. The second one [13-15] intends to protect the overused nodes against breakdown, aiming at maximizing the whole network lifetime.

\section{Proposed Schemes to Achieve Load Balancing}

A number of routing protocols proposed for MANETs use shortest route in terms of hop count for data transmission. It may lead to quick depletion of resources of nodes falling on the shortest route. It may also result in network congestion resulting in poor performance. Therefore, instead of hop count a new routing metric is required that can consider the node's current traffic and battery status while selecting the route. The idea is to select a routing path that consists of nodes with higher residual battery power and hence longer life.

We define the required parameters, as follows: The terms used in this paper have been defined as follows:

1) Route Energy (RE): The route energy of a path is the minimum of residual energy of nodes $\left(\mathrm{re}_{\mathrm{i}}\right)$ falling on a route. Higher the route energy, lesser is the probability of route failure due to exhausted nodes.

2) Traffic queue (tq): The traffic queue of a node is the number of packets queued up in the node's interface. Higher is its value, more occupied the node is.

3) Average Traffic Queue (ATQ): It is the mean of traffic queue of nodes from the source node to the destination node. It indicates load on a route and helps in determining the heavily loaded route.

4) Hop count (HC): The HC is the number of hops for a feasible path.

\subsection{Scheme 1}

The first scheme proposed in this paper tends to determine the routes in such a way that the routes consisting of nodes with lower residual battery capacity are avoided for data transmission even if they are short and less congested. This scheme tries to make a fair compromise between three route selection parameters i.e. hop count, residual battery capacity and traffic load.

A MANET can be represented as an undirected graph $\mathrm{G}(\mathrm{V}, \mathrm{E})$ where $\mathrm{V}$ is the set of nodes (vertices) and $\mathrm{E}$ is the set of links (edges) connecting the nodes. The nodes may die because of depleted energy source and the links can be broken at any time owing to the mobility of the nodes. $\forall \mathrm{n} \mid \mathrm{n} \epsilon \mathrm{V}, \mathrm{n}$ has an associated traffic queue $\mathrm{tq}(\mathrm{n})$ and residual battery energy $\mathrm{re}_{\mathrm{i}}$. A path between two 
nodes $\mathrm{u}$ and $\mathrm{v}$ is given as

$\mathrm{P}(\mathrm{u}, \mathrm{v})=(\mathrm{u}, \mathrm{e}(\mathrm{u}, \mathrm{x}), \mathrm{x}, \mathrm{e}(\mathrm{x}, \mathrm{y}), \mathrm{y}, \ldots \ldots . ., \mathrm{e}(\mathrm{z}, \mathrm{v}), \mathrm{v})$

It can be emphasized that a path between any two nodes is a set consisting of all possible paths between them. Formally, $\mathrm{P}(\mathrm{u}, \mathrm{v})=\left\{\mathrm{P}_{0}, \mathrm{P}_{1}, \ldots ., \mathrm{P}_{\mathrm{n}}\right\}$ where each $\mathrm{P}_{\mathrm{i}}$ is a candidate path between $\mathrm{u}$ and $\mathrm{v}$.

Let $\mathrm{HC}\left(\mathrm{P}_{\mathrm{i}}\right)$ be the hop count corresponding to path $\mathrm{P}_{\mathrm{i}}$ between $\mathrm{u}$ and $\mathrm{v}$. Weight of path $\mathrm{P}_{\mathrm{i}}$ defined as:

$$
\mathrm{W}\left(\mathrm{P}_{\mathrm{i}}\right)=\mathrm{W}_{1}{ }^{*} \mathrm{RE}\left(\mathrm{P}_{\mathrm{i}}\right)-\mathrm{W}_{2}{ }^{*} \operatorname{ATQ}\left(\mathrm{P}_{\mathrm{i}}\right)-\mathrm{W}_{3}{ }^{*} \mathrm{HC}\left(\mathrm{P}_{\mathrm{i}}\right)
$$

where $\operatorname{RE}\left(\mathrm{P}_{\mathrm{i}}\right)=\min \left\{\mathrm{re}_{\mathrm{n} 1}, \mathrm{re}_{\mathrm{n} 2}, \ldots, \mathrm{re}_{\mathrm{nm}}\right\}$ and $\mathrm{n}_{1}, \mathrm{n}_{2}, \ldots$, $\mathrm{n}_{\mathrm{m}}$ are the nodes making up the path.

$$
\operatorname{ATQ}\left(\mathrm{P}_{\mathrm{i}}\right)=\left(\operatorname{tq}\left(\mathrm{n}_{1}\right)+\operatorname{tq}\left(\mathrm{n}_{2}\right)+\ldots+\operatorname{tq}\left(\mathrm{n}_{\mathrm{m}}\right)\right) / \mathrm{m}-1
$$

The fields having adverse contribution to traffic distribution are built into negative coefficients in Equation (1). Also the weight values are calculated such that $\mathrm{W}_{1}+$ $\mathrm{W}_{2}+\mathrm{W}_{3}=1$.

The idea is to find a path from source to destination with maximum weight such that from the very beginning the path determined is energy efficient and there is a fair compromise between a short route and a light-loaded route. In this scheme $\mathrm{RE}$ has been given maximum weightage, i.e. $W_{1}$ is maximum and $W_{2}$ and $W_{3}$ are equal. We call this path Energy Aware Load-balanced Path (EALP).

Supposing that i $\epsilon\{0,1,2, \ldots, n\}, P(s, d)=\left\{P_{0}, P_{1}, \ldots, P_{n}\right\}$ for given source $\mathrm{s}$ and destination $\mathrm{d}$, we can define the problem mathematically as:

$$
\operatorname{EALP}(\mathrm{s}, \mathrm{d})=\mathrm{P}_{\mathrm{i}} \text { with }
$$

$$
\mathrm{W}\left(\mathrm{P}_{\mathrm{i}}\right)=\max \left\{\mathrm{W}\left(\mathrm{P}_{1}\right), \mathrm{W}\left(\mathrm{P}_{2}\right), \ldots, \mathrm{W}\left(\mathrm{P}_{\mathrm{n}}\right)\right\}
$$

$\mathrm{W}_{1}, \mathrm{~W}_{2}$ and $\mathrm{W}_{3}$ are constants.

In proposed scheme routes are determined on demand. A source node initiates the route discovery process by broadcasting a route request (RREQ) packet whenever it wants to communicate with another node for which it has no routing information in its table. On receiving a RREQ packet, a node checks its routing table for a route to the destination node. If the routing table contains the latest route to the destination node, the intermediate node sends a RREP packet along the reverse path back to the source node also appending the weight value for the route. When a source node receives more than one RREP packet for a RREQ, it compares the weight values of the routes and selects the route with maximum weight. However, if an intermediate node has no information of the destination node, it adds its own traffic queue value, compares and finds the minimum of residual battery capacity field of RREQ packet with its own residual battery capacity and updates residual battery capacity field of RREQ packet, increments the hop count by one and rebroadcasts the route discovery packet. When destination node receives a route request packet, it waits for a certain amount of time before replying with a RREP packet in order to receive other RREQ packets. Then destination node computes ATQ and the weight value for each fea- sible path using Equation (2) and using weight function as given in Equation (1) respectively. The route with highest weight value is selected as the routing path and a RREP packet is sent back towards the source node on the selected path.

In the algorithm discussed above weight values are constant, which is its limitation as when route selection procedure starts there are more chances of network congestion because of flooding of many RREQ packets simultaneously. Moreover, nodes have maximum battery energy during initial phases. Therefore, the requirement is to change the above algorithm such that when the battery energy of nodes is high, emphasis is on selecting a short and light loaded route. As battery energy of nodes decreases we tend to conserve energy, compromising on short and lightly loaded route.

\subsection{Scheme 2}

Another scheme has been proposed in this paper in which weight values $\left(\mathrm{W}_{1}, \mathrm{~W}_{2}\right.$ and $\left.\mathrm{W}_{3}\right)$ are adaptive to the network status, instead of being constant. More weight age is given to find short and less congested routes during initial route discovery procedure, as the possibility of network congestion is high due to flooding of many RREQ packets simultaneously. Also, nodes have maximum battery energy during initial phases. However, as the time elapses battery energy of nodes decreases, therefore, we tend to conserve energy, compromising on short and lightly loaded routes. The adaptive behavior of the protocol has been implemented by computing the proportion of route energy and initial energy of nodes assuming that all nodes are similar with equal initial battery energy. Therefore, as per Scheme 2, weight value of a route is computed as:

$$
\mathrm{W}\left(\mathrm{P}_{\mathrm{i}}\right)=(1-\alpha) * \operatorname{RE}\left(\mathrm{P}_{\mathrm{i}}\right)-\alpha / 2 *\left(\mathrm{ATQ}\left(\mathrm{P}_{\mathrm{i}}\right)+\mathrm{HC}\left(\mathrm{P}_{\mathrm{i}}\right)\right),
$$

where,

$$
\alpha=\min \left(\operatorname{RE}\left(\mathrm{P}_{\mathrm{i}}\right)\right) / \mathrm{IE} ; 0 \leq \alpha \leq 1
$$

and gives the proportion of battery capacity left. Initially when nodes have high residual battery energy $\alpha$ is maximum, route selection is mainly done on the basis of hop count and average traffic load as can be seen from Equation (4). As nodes battery energy decreases with the passage of time $\alpha$ decreases and 1- $\alpha$ increases leading to more weightage to the route energy parameter.

\subsection{Scheme 3}

The scheme proposed next uses location information to limit the broadcast of RREQ packets. When an intermediate node receives a RREQ packet it uses the location information before broadcasting the RREQ packets further. Only the nodes that are closer to the destination than the source node are allowed to broadcast RREQ 
packets further. By doing so a broadcast storm can be avoided resulting in less congested routes. Flowchart given in Figure 3 gives the details of this algorithm.

A source node while starting a route discovery process, computes its distance w.r.t. the destination node, appends this value in the RREQ packet along with the fields as used in Scheme 2 and broadcasts it further. An intermediate node on receiving a RREQ packet, compares its distance to the destination node with the distance value stored in the RREQ packet. If its distance is longer, it drops the RREQ packet else it compares the energy value in the record of the RREQ packet with its own energy and assigns the lesser energy as the new energy value in the packet. It also adds its own traffic queue to the traffic queue already recorded in the packet and updates hop count by 1 . It then broadcasts the packet further. By doing so only those nodes that are closer to the destination node than the source node participate in route selection procedure resulting in reduced routing overhead. This procedure has been explained with the help of Figures 1 and 2.

\subsection{Example}

As shown in Figure 1, we assume that there are three feasible paths from source node $\mathrm{S}$ and destination node $\mathrm{D}$ - Path I: (S,A,E,H,J,D), Path II: (S,B,F,K,D), Path III: (S,C,G,I,L,M).

Corresponding to Figure 1, the nodes on Path I (S,A,

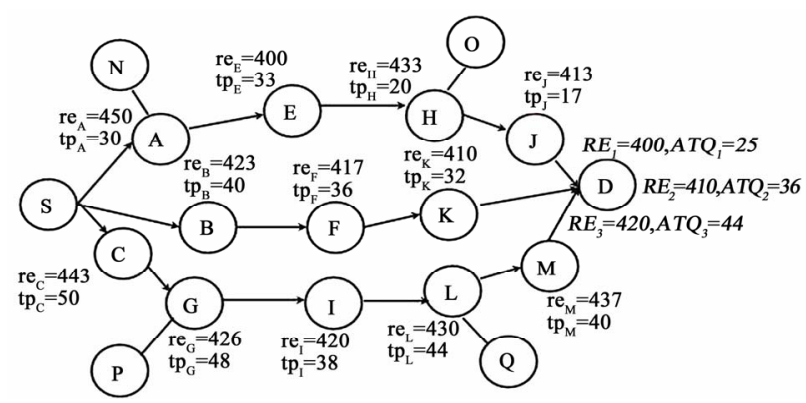

Figure 1. Route energy and average traffic queue of each feasible path for high residual battery capacity of nodes.

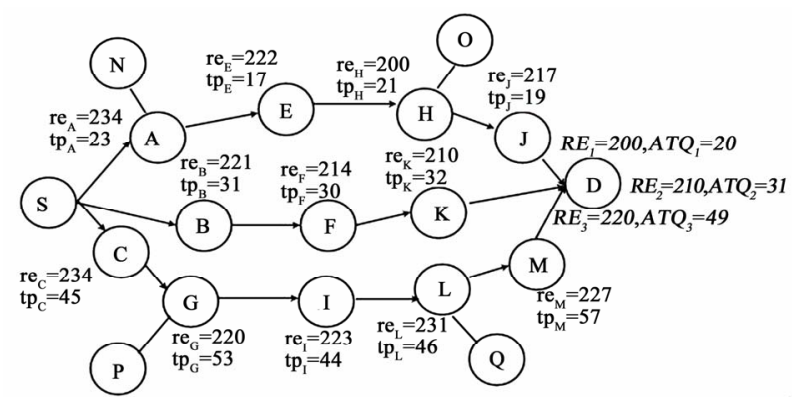

Figure 2. Route energy and average traffic queue of each feasible path for low residual battery capacity of nodes.
E,H,J,D), energies of intermediate nodes between source and destination are $(450,400,433,413)$; thus $\mathrm{RE}_{1}=$ $\min (450,400,433,413)=400$. Similarly, for Path II $\mathrm{RE}_{2}=410$ and for Path III $\mathrm{RE}_{3}=420$.

The traffic queue length of all the intermediate nodes between source and the destination as shown in Figure 1, for Path I (S,A,E,H,J,D), $\mathrm{ATQ}_{1}=25, \mathrm{HC}_{1}=5$. For Path II $(\mathrm{S}, \mathrm{B}, \mathrm{F}, \mathrm{K}, \mathrm{D}), \mathrm{ATQ}_{2}=36, \mathrm{HC}_{2}=4$ and for Path III $(\mathrm{S}, \mathrm{C}, \mathrm{G}, \mathrm{I}, \mathrm{L}, \mathrm{M}), \mathrm{ATQ}_{3}=44, \mathrm{HC}_{3}=6$.

The destination node on receiving a RREQ packet waits for certain amount of time before replying with a RREP packet in order to receive more RREQ packets. According to first scheme the weight values are constant. After performing many simulations, we have determined that we get most favorable results for $\mathrm{W}_{1}=0.6, \mathrm{~W}_{2}=\mathrm{W}_{3}=$ 0.2 . On substituting these weight values and parameters as described above in Equation (1) we get $\mathrm{W}_{3}>\mathrm{W}_{2}>\mathrm{W}_{1}$. Hence, Path III is the most suitable route and hence is selected for data transmission.

For the other two schemes we compute the value of $\alpha$ as per Equation (5). On substituting $\alpha$ in Equation (1), we get $\mathrm{W}_{1}>\mathrm{W}_{2}>\mathrm{W}_{3}$ i.e. initially when the nodes have high residual battery capacity, more weightage is given to the short and lightly loaded route while route selection. However, a trade-off between hop count and ATQ is still maintained in order to avoid congested routes. As the battery energy of nodes diminishes, more emphasis is given on selecting the routes with high residual battery power. Although the routes selected may be longer. In this situation, Scheme 1 still results in $\mathrm{W}_{3}>\mathrm{W}_{2}>\mathrm{W}_{1}$, however, for Schemes 2 and 3, the weight values have changed from $\mathrm{W}_{1}>\mathrm{W}_{2}>\mathrm{W}_{3}$ for high residual battery capacity to $\mathrm{W}_{3}>\mathrm{W}_{2}>\mathrm{W}_{1}$ for low energy nodes. This comparison has been illustrated in Table 1 and Table 2 .

Table 1. Comparison of schemes for high vales of route energy.

\begin{tabular}{cccccc}
\hline \multirow{2}{*}{ Path } & $\begin{array}{c}\text { RE } \\
\text { (IE=500) }\end{array}$ & ATQ & HC & \multicolumn{2}{c}{ Route weight } \\
\cline { 4 - 6 } & & & Scheme 1 & Scheme 2 \& 3 \\
\hline P1 & 400 & 25 & 5 & & \\
P2 & 410 & 36 & 4 & $\mathrm{~W}_{3}>\mathrm{W}_{2}>\mathrm{W}_{1}$ & $\mathrm{~W}_{1}>\mathrm{W}_{2}>\mathrm{W}_{3}$ \\
P3 & 420 & 44 & 6 & & \\
\hline
\end{tabular}

Table 2. Comparison of schemes for low values of route energy.

\begin{tabular}{cccccc}
\hline Path & $\begin{array}{c}\text { RE } \\
(\text { IE=500) }\end{array}$ & ATQ & HC & \multicolumn{2}{c}{ Route weight } \\
\cline { 5 - 6 } & & & & Scheme 1 & Scheme 2 \& 3 \\
\hline P1 & 200 & 20 & 5 & & \\
P2 & 210 & 31 & 4 & $\mathrm{~W}_{3}>\mathrm{W}_{2}>\mathrm{W}_{1}$ & $\mathrm{~W}_{3}>\mathrm{W}_{2}>\mathrm{W}_{1}$ \\
P3 & 220 & 49 & 6 & & \\
\hline
\end{tabular}




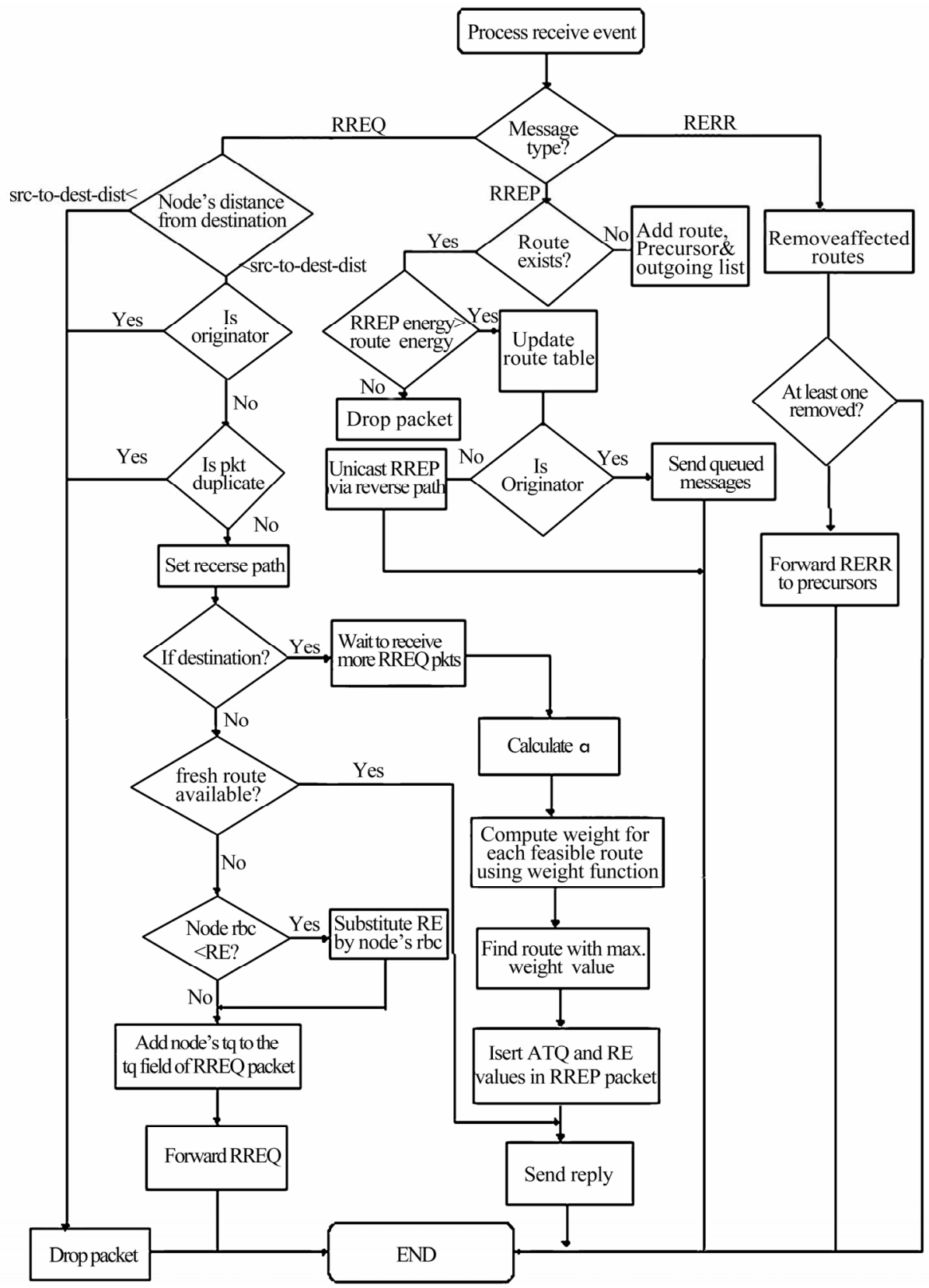

Figure 3. Flowchart depicting proposed algorithm.

\section{Performance Evaluation}

In this section we describe our simulation environment and performance metrics.

\subsection{Performance Metrics}

We have used ns-2 simulator version 2.29 to analyze the proposed algorithms. Our solution has been compared against AODV and two of the previously proposed load balanced ad hoc routing protocols - DLAR and LARA.
We use the following performance metric to evaluate the performance of each scheduling algorithm:

- Packet Delivery Fraction: It gives the ratio of the data packets delivered to the destination to those generated by the sources, which reflects the degree of reliability of the routing protocol.

- Normalized Routing Load: The number of routing control packets per data packet delivered at the destination.

- Average End-to-End Delay: This is the average overall delay for a packet to traverse from a 
source node to a destination node. This includes the route discovery time, the queuing delay at a node, the transmission delay at the MAC layer, and the propagation and transfer time in the wireless channel. As delay primarily depends on optimality of path chosen, therefore, this is a good metric for comparing the efficiency of underlying routing algorithms.

- Jitter: Jitter is defined as the delay variation between each received data packets. It gives an idea about stability of the routing protocol.

- Average Residual Battery Capacity: This metric depicts the amount of energy consumption of nodes with respect to time period.

\subsection{Simulation Environment}

Our simulation scenario consists of 50 nodes moving at maximum velocity of $20 \mathrm{~m} / \mathrm{s}$ in a $600 \mathrm{~m} \times 600 \mathrm{~m}$ grid area with a transmission range of $100 \mathrm{~m}$ with 25 and 37 TCP flows. Each source node transmits packets at a rate of four packets per second, with a packet size of 1024 bytes. We run simulation for pause times of $0,100,200,300$, 400, 500, 600, 700 and 900 seconds. The mobility of a node is defined by random waypoint model. This model forces nodes to move around with two predefined parameters, maximum velocity and pause time. Each node moves to a random destination at random velocity. They stay there for predefined time and then move to a new destination. Also it is the most widely used mobility model in previous studies. The size of the interface buffer of each node for simulation is taken as 50 packets. Each experiment is conducted four times and the average result has been considered.

\subsection{Simulation Results}

\subsubsection{Packet Delivery Fraction}

Figure 4 and Figure 5 show the packet delivery fraction of each protocol for 50 nodes with 25 and 37 sources respectively. The proposed schemes perform very well irrespective of the node's pause time and outperform AODV, DLAR and LARA. In high mobility scenarios, many route construction processes are invoked. When a source floods a RREQ packet to recover the broken route, many intermediate routes reply with the routes cached by overhearing packets during the initial route construction phase. A number of these cached routes overlap existing routes. Nodes that are part of multiple routes become congested and can not deliver the packets further resulting in poor performance of AODV. Although DLAR and LBAR also achieve a better performance than AODV, the effectiveness of load balancing is not salient compared

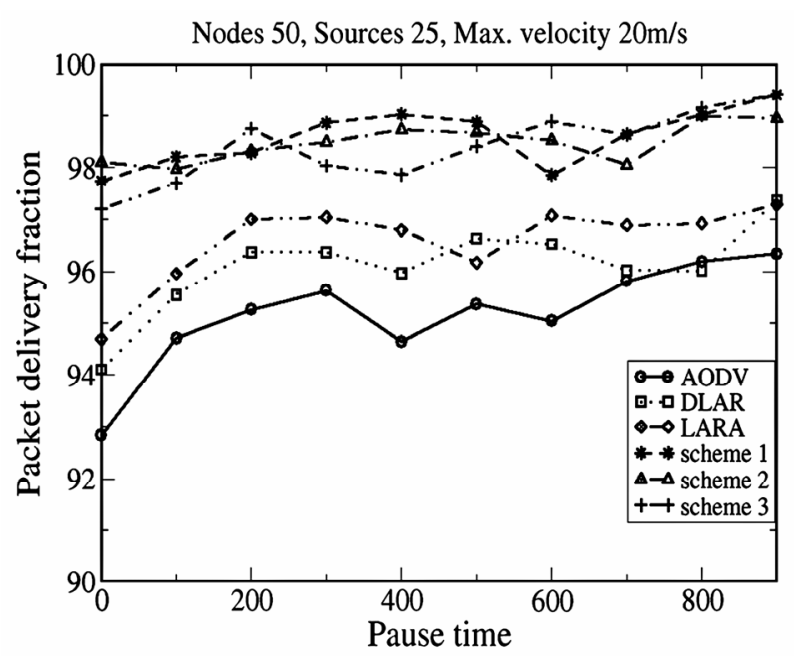

Figure 4. Packet delivery fraction vs. pause time for 25 sources.

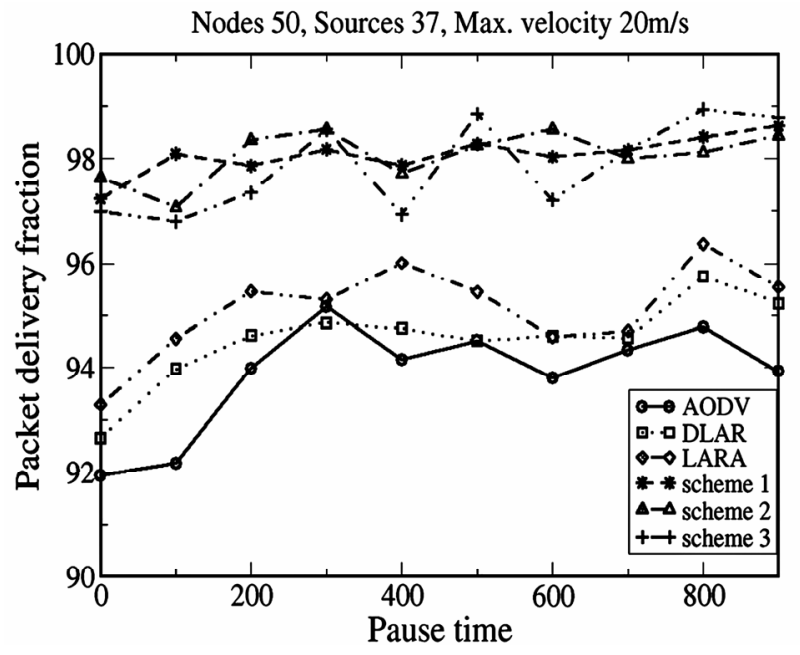

Figure 5. Packet delivery fraction vs. pause time for 37 sources.

with our schemes. The performance of proposed schemes is almost similar. However, the reason for lower packet delivery fraction at some points for third scheme is inability of the network to find out a route to the destination because of restricted number of RREQ packets. The results also show that the packet delivery fraction reduces with increase in load in the network.

\subsubsection{Normalized Routing Load}

Figure 6 and Figure 7 show normalized routing load of each protocol for 50 nodes with 25 and 37 sources respectively. Horizontal axis of the figures represent the pause times. As expected, normalized routing load for first two proposed schemes is comparatively higher than AODV protocol. However, in the third proposed algorithm 


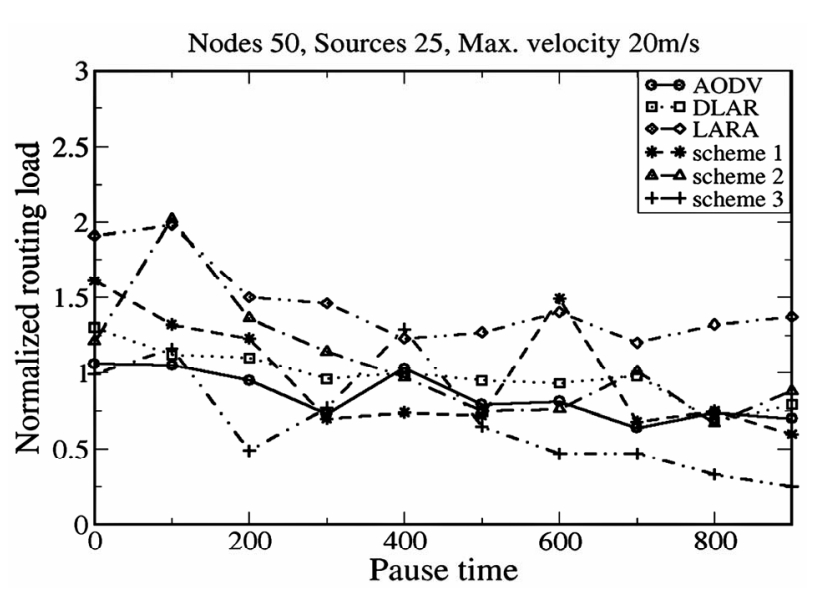

Figure 6. Normalized routing load vs. pause time for 25 sources

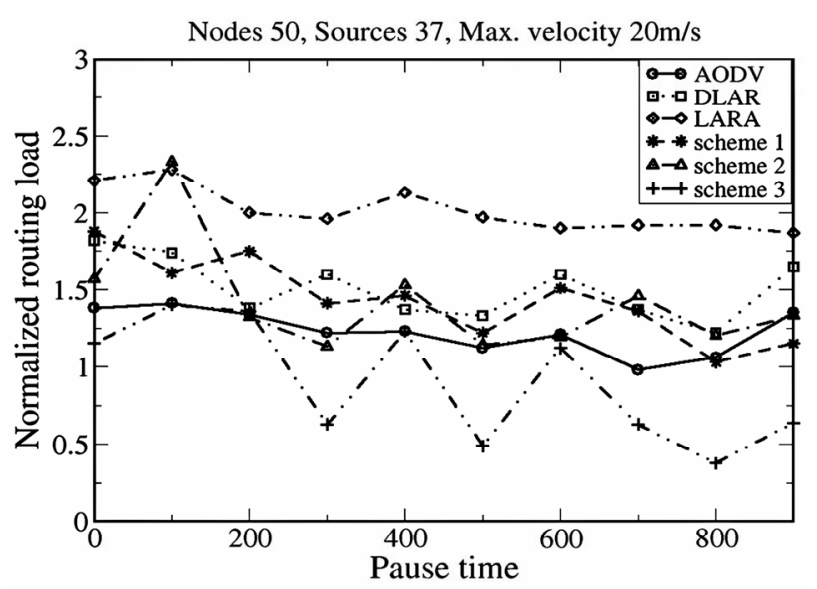

Figure 7. Normalized routing load vs. pause time for 37 sources.

we try to restrict the broadcast of RREQ packets, which results in lower routing load than the routing load of AODV, DLAR and LARA protocols. It has also been observed from Figure 6 and Figure 7 that normalized routing load increases with increase in number of sources in the network.

\subsubsection{Average End-to-End Delay}

Figure 8 and Figure 9 plot the average end-to-end delay for variations of node's pause time for 50 nodes with 25 and 37 sources respectively. Proposed algorithms have much improved average end-to-end delay than AODV and other two load balanced routing protocols i.e. DLAR and LARA. We can see that the end-to-end delay increases for all the protocols with increase in load as can be seen in Figures 8 and 9. The reason is the increased contention at MAC level due to increase in load. The packets now have to wait longer in the interface queue before being transmitted. Here, AODV suffers maximum delay as it often routes the packets around heavily loaded nodes. DLAR and LARA make better choice of routes than AODV. The proposed algorithms make best decision among all these protocols. The results are more noteworthy because even for highly dynamic topology (i.e. pause time $=0$ ) and static topology (i.e. pause time $=900$ ), proposed algorithms achieve significantly lower delay than rest three protocols. This is due to the effective routing strategy adopted for load balancing and their try to route packets along a less congested route to avoid overloading of some nodes.

\subsubsection{Jitter}

Figure 10 and Figure 11 show delay variation of received packets (jitter) versus pause time for 50 nodes with 25 and 37 sources respectively. It can be seen that jitter is considerably lower for proposed algorithms than AODV DLAR and LARA protocols, even for highly dynamic

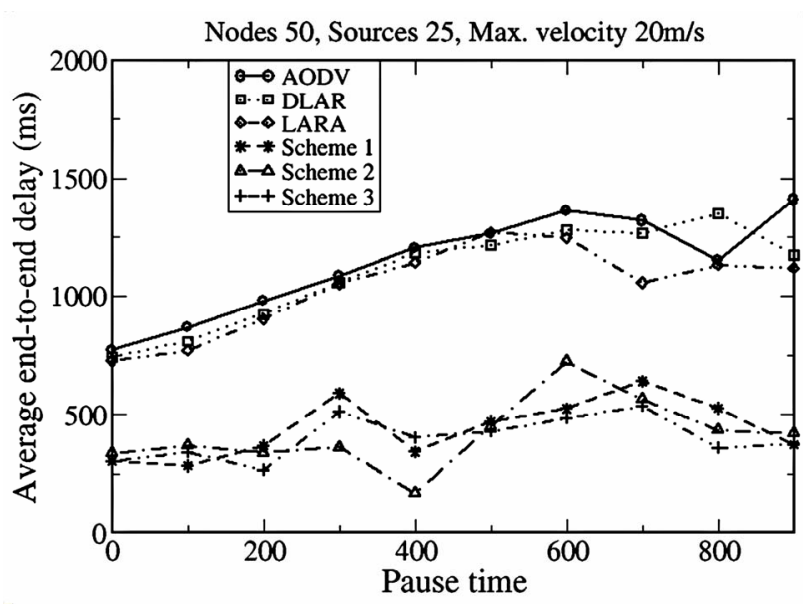

Figure 8. Average end-to-end delay vs. pause time for 25 sources.

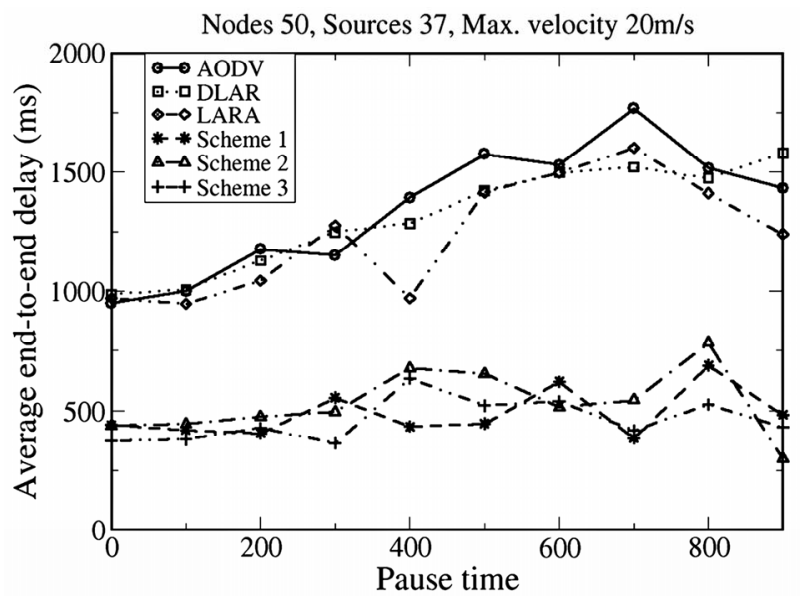

Figure 9. Average end-to-end delay vs. pause time for 37 sources. 
topology (i.e. pause time $=0$ ) and nearly static topology (i.e. pause time $=900$ ) as well. This behavior is as anticipated because delays mainly occur in queuing and medium access control processing. These delays are reduced in proposed schemes by routing the packets towards nodes that are less occupied also taking into account more efficient nodes in terms of energy.

\subsubsection{Average Residual Battery Capacity}

Figure 12 compares the average residual battery capacity of nodes for AODV and the proposed schemes w.r.t. simulation time. It is evident from the figure that the rate of energy consumption is much higher for AODV than the proposed protocols. The reason is the energy aware load balancing behavior of proposed schemes. Initially when battery energy of nodes is high, energy consumption rate for the first proposed scheme is the least. This is due its behavior of energy considerations while balancing the load, even if the node energy is high. The performance of other two protocols improves with the reduc-

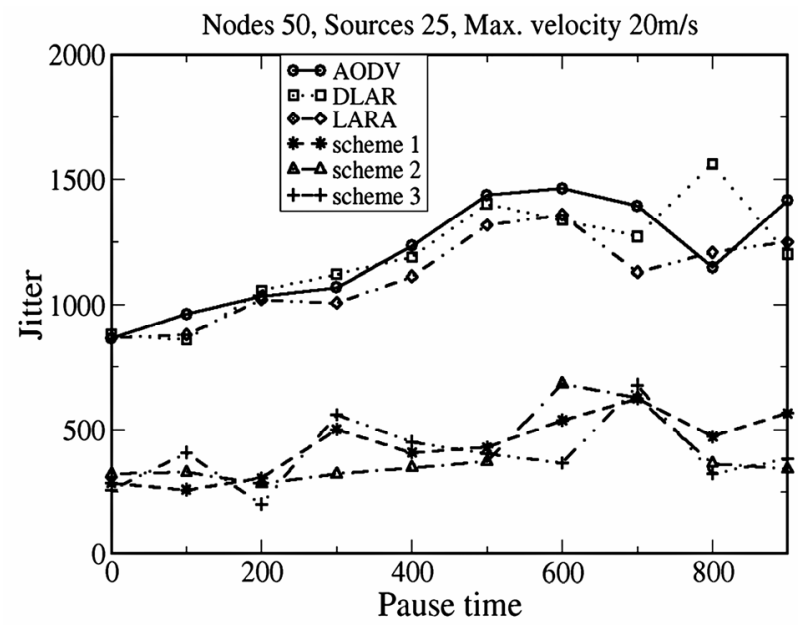

Figure 10. Jitter vs. pause time for 25 sources.

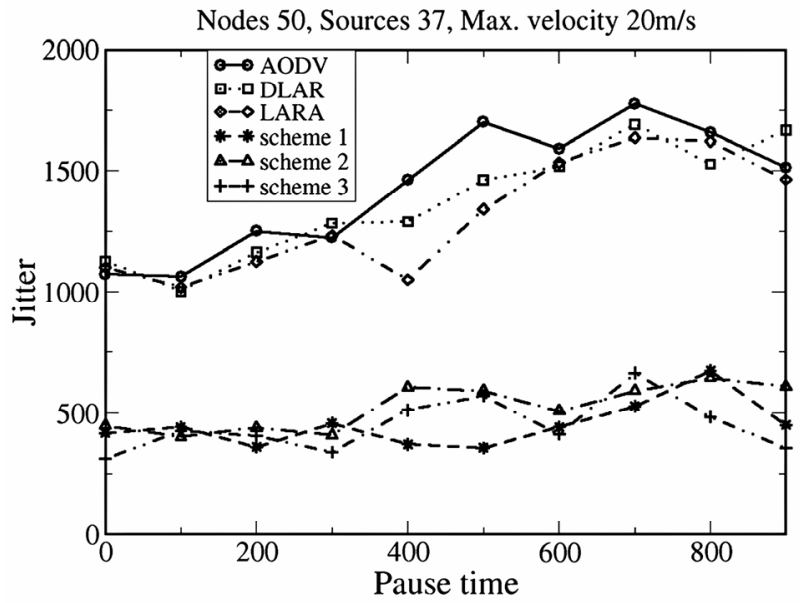

Figure 11. Jitter vs. pause time for 37 sources.

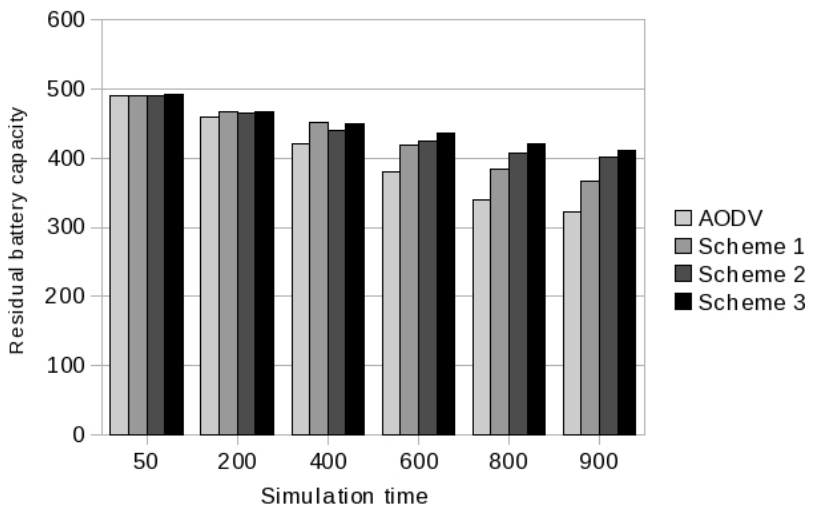

Figure 12. Average residual battery capacity of nodes.

tion in battery energy, because as the battery capacity of nodes decreases, routes with higher residual battery capacity are considered irrespective of its length and load. As can be inferred from the Figure 12, a MANET employing third proposed strategy for routing has maximum residual battery capacity. It is due to restricting the broadcast of packets. As a result of which a proportion of energy spent by nodes in forwarding RREQ packets remains conserved.

\section{Conclusions}

In this paper, we presented some schemes for load balancing in mobile ad hoc networks. The proposed schemes are based on a new metric based on weighted combination of three parameters. The three parameters responsible for final route selection are - the average traffic queue, the route energy, and the hop count. And, the weights corresponding to these parameters may be fixed or adaptive to the network status, depending upon the load balancing scheme. By taking these three parameters together the traffic is deviated from high loaded routes towards routes possessing higher energy and less loaded. In proposed strategies a load balanced routing path is selected among all feasible paths on the basis of weight value calculated for each path. In a feasible path, the higher the weight value, the higher is its suitability for traffic distribution. The performance of the schemes is evaluated by simulation. The result of simulation indicates that, compared with previous load balanced routing schemes DLAR and LBAR, the proposed schemes exhibit a better performance in both moderately loaded and highly loaded situations. In addition, we have shown that the average residual battery capacity of nodes and hence network lifetime is higher in case of proposed schemes than AODV protocol.

\section{References}

[1] C. E. Perkins and E. M. Royer, and S. R. Das, "Ad hoc 
on-demand distance vector routing," Internet Draft, draft-ietf-manet-aodv-05.txt, March 2000.

[2] D. B. Johnson and D. A. Maltz, "The dynamic source routing protocol for mobile ad hoc networks," IETF Draft, 1999.

[3] S. J. Lee and M. Gerla, "Dynamic load aware routing in ad hoc networks," Proc. ICC, Helinski, Finland, pp. 3206-3210, June 2001.

[4] V. Saigal, A. K. Nayak, S. K. Pradhan, and R. Mall, "Load balanced routing in mobile ad hoc networks," Elsevier Computer Communications, Vol. 27 pp. 295-305, 2004,.

[5] H. Hassanein and A. Zhou, "Routing with load balancing in wireless ad hoc networks," Proc. ACM MSWiM, Rome, Italy, pp. 89-96, July 2001.

[6] K. Wu and J. Harms, "Load sensitive routing for mobile ad hoc networks," Proc. IEEE ICCCN, Phoenix, AZ, pp. 540-546, Oct. 2001.

[7] J.-W. Jung, D. I. Choi, K. Kwon, I. Chong, K. Lim, and H.-K. Kahng, "A correlated load aware routing protocol in mobile ad hoc networks," ECUMN, LNCS 3262, pp. 227-236, 2004.

[8] M. R. Pearlman, Z. J. Hass, P. Sholander, and S. S. Tabrizi, "On the impact of alternate path routing for load balancing in mobile ad hoc networks," Proc. of First Annual Workshop on Mobile and Ad Hoc Networking and Computing, Mobihoc, Boston, MA, USA, pp. 3-10, August 2000 .

[9] A. Rani and M. Dave, "Performance evaluation of modi- fied AODV for LOAD balancing," Journal of Computer Science, Vol. 3, pp. 863-868, 2007.

[10] S. Singh, M. Woo, and C. Raghavendra, "Power-aware routing in mobile ad-hoc networks," Proceedings of the 4th Annual ACM/IEEE International Conference on Mobile Computing and Networking (MobiCom), Dallas, TX, USA. New York, NY, pp. 181-190, Oct 25-30, 1998.

[11] A. Srinivas and E. Modiano, "Minimum energy disjoint path routing in wireless ad-hoc networks," Proceedings of the 9th Annual International Conference on Mobile Computing and Networking (MobiCom), San Diego, CA, USA. New York, NY, pp. 122-133, Sep 14-19, 2003.

[12] M. Subbarao, "Dynamic power-conscious routing for manets: An initial approach," Proceedings of the 50th IEEE Vehicular Technology Conference, VTC, Vol. 2, pp. 1232-1237, Sep 19-22, 1999.

[13] C. K. Toh, "Maximum battery life routing to support ubiquitous mobile computing in wireless ad-hoc networks," IEEE Communications Magazine, Vol. 39, pp. 138-147, 2001.

[14] N. Gupta and S. R. Das, "Energy-aware on-demand routing for mobile ad-hoc networks," Proceedings of the 4th International Workshop on Distributed Computing, IWDC, Capri, Italy, pp. 164-173, Sep 8-11, 2002.

[15] L. Y. Li, C. L. Li, and P. Y. Yuan, "An energy level based routing protocol in Ad-hoc networks," Proceedings of the IEEE/WIC/ACM International Conference of Intelligent Agent Technology (IAT'06), Hong Kong, China. Los Alamitos, CA, pp. 306-313, Dec 18-22, 2006. 